\title{
Challenges and controversies in thoracic oncology: a new ERJ series
}

\author{
Jean-Paul Sculier ${ }^{1}$, Benjamin Besse ${ }^{2}$ and Paul Van Schil ${ }^{3}$ \\ Affiliations: ${ }^{1}$ Service des soins intensifs et urgences oncologiques \& Oncologie thoracique, Institut Jules \\ Bordet, Université Libre de Bruxelles, Brussels, Belgium. ${ }^{2}$ Dept of Cancer Medicine/Thoracic Unit, Institut \\ Gustave Roussy, Villejuif, France. ${ }^{3}$ Dept of Thoracic and Vascular Surgery, Antwerp University Hospital, \\ Edegem, Belgium.
}

Correspondence: J-P. Sculier, Institut Jules Bordet, 1 rue Héger-Bordet, Bruxelles, B-1050, Belgium.

E-mail: sculierabordet.be

@ERSpublications

This month sees the launch of a new review series in the ERJ, which will cover the hottest topics in lung cancer http://ow.ly/uZPjD

Appearing in the present issue of European Respiratory Journal is the first article of a new series dealing with challenges and controversies in thoracic oncology. The uniqueness of the series lies in the multidisciplinary approach to the choice of the topics and authors. Indeed, thoracic oncology, which mainly deals with the most important cancer in term of mortality, is involved in many medical disciplines: epidemiology, pathology, biology, chest medicine, imaging, medical oncology, radiation oncology, surgical oncology, and so on. Care proposals are today decided in multidisciplinary conferences where all those specialties should be represented and where the chest physician has a key role [1-3]. In addition, our knowledge about malignant diseases of the chest, whether common or rare, has substantially improved, particularly in the biology field, leading to the identification of therapeutic targets for which the first routine applications are available.

All the progress made in these different disciplines can result in decision conflicts, because we often lack good evidence for performing the best choice. Indeed, during the past decade, if academic research has provided very good data in translational research, epidemiology and diagnostic procedures, then randomised clinical trials have practically become the monopoly of the industry, which favours clinical research where immediate profits can be made. For example, the curative effect of adjuvant chemotherapy after lung cancer resection has been established by academic trials that required a long period of time for accrual and follow-up before analysis. Today, in 2014, in the context of the new European legislations associated with increased bureaucracy and a high cost, the investigation of such a question by multiple independent randomised trials will not be possible, leaving the topic at the conceptual stage and without possible routine implementation.

The role of the present series published in the European Respiratory Journal is to cover some of the present difficult topics and controversies in thoracic oncology. The specific contribution of each thoracic staging and treatment modality has not always been unequivocally established and many grey areas remain. Concerning screening programmes for lung cancer, is it time for large-scale screening via chest computed tomography? Is multidisciplinary team management in thoracic oncology more than a concept? Considering tumour tissue sampling for lung cancer management at the time of personalised therapy, what is enough for molecular testing? Which approaches for small sized lung cancer: lobectomy, sublobectomy or stereotactic irradiation? What is the place today of the multimodality management of malignant pleural mesothelioma? How can non-inferiority trials influence practice in thoracic oncology without valid conclusions? How should advanced non-small cell lung cancer (NSCLC) be treated: by target 
therapy, personalised chemotherapy or standard chemotherapy? What is the exact role of palliative care in the patient's management? What should be the approach to the staging of lung cancer, with regard to noninvasive, minimally invasive and invasive techniques? Which examinations (and at what time) should take place during the follow-up of the patient after lung cancer resection? What is the best management option for a patient with NSCLC, with an EGFR activating mutation or with an Alk translocation?

Our purpose is not to be exhaustive but to cover some of the hottest topics that we meet in our daily practice. The authors have been selected for their expertise and come from various specialties. This approach follows the objectives of the action plan to make thoracic oncology one the pillars of the European Respiratory Society [4].

\section{References}

1 Gaga M, Powell CA, Schraufnagel DE, et al. An official American Thoracic Society/European Respiratory Society statement: the role of the pulmonologist in the diagnosis and management of lung cancer. Am J Respir Crit Care Med 2013; 188: 503-507.

2 Gaga M, Sculier JP, Rabe KF. Pulmonologists and lung cancer: pivotal role in multidisciplinary approach. Eur Respir J 2013; 42: 1183-1185.

3 Blum TG, Rich A, Baldwin D, et al. The European initiative for quality management in lung cancer care. Eur Respir J 2014; 43: 1254-1277.

4 Sculier JP, Vansteenkiste J, Schonfeld N, et al. Thoracic oncology in Europe: the ERS action plan by the Thoracic Oncology Assembly. Eur Respir J 2010; 36: 1227-1228. 